\title{
A temible complication of ischemic stroke: pulmonary embolism
}

\section{Abstract}

Stroke is a leading cause of death and disability and is the third highest cause of death in the western world. Medical illness after ischemic stroke contribute substantially to poor stroke outcomes. The early in-hospital complications of stroke patients are mainly: pneumonia (hospital acquired pulmonary infection), increased intracranial pressure (neuroimaging evidence of cerebral edema or brain shift syndrome with clinical deterioration), urinary tract infection, intracerebral bleeding, recurrent stroke, epileptic seizures, thrombosis or pulmonary embolism. All these complications influence the patient's outcomes and require diagnostic and therapeutic measures.

Pulmonary embolism is a major contributor to in-hospital death after stroke. Although the rate of clinically overt pulmonary embolism afterstroke has been estimated to be less than 1\%, pulmonary emboli account for up to 50\% of early deaths after stroke. In daily practice, the clinical burden of pulmonary embolism in patient with stroke is, however, underestimated since the clinical symptoms of stroke may obscure the recognition of this complication. The aim of this article is to describe the clinical and therapeutic aspects of pulmonary embolism as complication after stroke.

Keywords: Ischemic stroke; Pulmonary embolism; Complications

Una temibile complicanza dell'ictus ischemico: l'embolia polmonare

CMI 2015; 9(4): 101-108

http://dx.doi.org/10.7175/cmi.v9i4.1196

\section{INTRODUCTION}

Pulmonary embolism (PE) is a widespread and life-threatening condition, representing the second cause of sudden death [1]: in fact, most patients affected by pulmonary embolism die within the first few hours from the event. Although several diagnostic advances have been made in recent years, delays in the diagnosis are common and represent an important limit. Frequently, the diagnosis of $\mathrm{PE}$ is missed due to non-specific signs and symptoms. If left untreated, about one-third of patients who survive an initial pulmonary embolism die from a subsequent embolic episode.
When a case of PE is recognized, it has to be classified as acute or chronic. In terms of pathologic diagnosis, an embolus is acute if it is situated centrally within the vascular lumen or if it occludes a vessel. Acute PE commonly causes distention of the involved vessel. An embolus is chronic if it is eccentric and contiguous with the vessel wall and reduces the arterial diameter by more than $50 \%$, and evidence of recanalization within the thrombus is present.

$\mathrm{PE}$ is also categorized as central or peripheral, depending on the location of the arterial branch involved. The aim of this article is to focus on pulmonary embolism as complication of ischemic stroke.

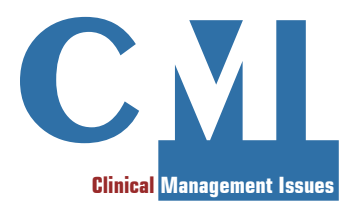

Clinical management
${ }^{1}$ U.O.C. di Neurologia,

ASP Caltanissetta Presidio

Ospedaliero Sant'Elia

${ }^{2}$ Direttore U.O.C.

di Neurologia

ASP Caltanissetta

P.O. Sant'Elia
Corresponding author

Dr Linda Iurato

lindaiurato@yahoo.it

Disclosure

The authors declare they have no conflict of interests regarding the publication of this article 
RISK ASSESSMENT OF DEEP-VEIN THROMBOSIS AND EPIDEMIOLOGY OF PE IN ISCHEMIC STROKE

$\mathrm{PE}$ is an uncommon but serious medical complication after an acute ischemic stroke (AIS). An effective thromboprophylaxis and an early diagnosis and treatment of venous thrombosis embolism (VTE) have been shown to reduce effectively the morbidity and mortality from $\mathrm{PE}$. In fact, $\mathrm{PE}$ is characterized by non-specific signs and symptoms, resulting in a correct and timely diagnosis just in a small percentage of patients who manifest objective symptoms. Especially elderly patients are often underdiagnosed and misdiagnosed. No reliable clinical signs and symptoms have been identified to make a definitive clinical diagnosis of DVT: when ancillary investigations enable the diagnosis, generally the patients are asymptomatic.

PE generally arises from venous thromboembolism developing in a paralyzed lower extremity after a stroke. The overall prevalence of clinically evident deep vein thrombosis (DVT) after acute stroke is around $2-20 \%$ and it develops mainly between two and seven days after the event [2].It can lead to post-thrombotic leg and varicose ulcers in addition to delaying rehabilitation.

The most powerful tool to lower the risk of DVT is prevention, feasible using maneuvers like:

- early mobilization;

- administration of antithrombotic agents; and

- the use of external compression device.

Factors with positive predictive value of DVT poststroke are:

- female gender;

- older age;

- body mass index $(\mathrm{BMI}) \geq 25 \mathrm{~kg} / \mathrm{m}^{2}$;

- cancer;

- hemorrhagic subtype stroke; and

- a lower limb NIHSS score $\geq 2$ [2].

In fact, obesity may restrict venous return secondary to body fat, preventing an efficient blood flow. In addition, fatty tissue has a proinflammatory, prothrombotic, and hypofibrinolytic role. This is why the risk of DVT is directly proportional to body weight.

The detection of an increased occurrence in patients affected by hemorrhagic stroke subtype may be attributed to the lack of antithrombotic usage after a hemorrhagic stroke.
Dehydration in acute stroke patients and the severity of leg weakness may also increase the risk of post-stroke DVT.

The annual incidence rate of $\mathrm{PE}$ in the general population is 0.50 to 0.69 per 1000 persons [3]; the mortality is high, ranging between $8.6 \%$ and $17 \%$ at 3 months. Immobility, older age, smoking, hypertension, thrombophilia, and cancer are commonly reported risk factors of $\mathrm{PE}$ and they are also frequent in AIS patients.

Pongmoragot and colleagues have described the clinical characteristics, risk factors and relevant clinical outcomes in patients who developed a PE within 30 days after an AIS [3]. Almost one-third of these patients died in the hospital after AIS and had higher rates of disability, higher case fatality at 30 days and 1 year, higher prevalence of in-hospital complications and only 1 out of 5 patients were discharged home. The risk of PE in AIS patients was higher in older patients with more severe stroke, history of cancer or DVT/PE or DVT during admission in stroke unit. PE was more frequent in cardioembolic and large artery atherosclerosis subtype stroke [3]. In fact, stroke subtypes are: lacunar, cardioembolic, large artery atherosclerosis, other, cryptogenic stroke.

\section{DIAGNOSIS OF PE}

As reported in the PIOPED study, patients with acute PE often present with dyspnea or chest pain (Table I).

Pulmonary embolism may present with a variety of symptoms, ranging from sudden catastrophic hemodynamic collapse (often observed in patients with prior poor cardiopulmonary status) to gradually progressive dyspnea.

Patients with PE may also present with atypical symptoms, such as:

- seizures;

- syncope;

- abdominal pain;

- fever;

- productive cough;

- wheezing;

- decreasing level of consciousness;

- new onset of atrial fibrillation;

- flank pain; and

- delirium (in elderly patients) [1]. 
If pulmonary infarction occurs, patients may also experience pleuritic chest pain with hemoptysis. The possibility of massive $\mathrm{PE}$ should be considered in patients who have a sudden onset of syncope, hypotension, extreme hypoxemia, electromechanical dissociation, or cardiac arrest.

Strong suspicion of $\mathrm{PE}$ is based on the presence of risk factors that lead to consider $\mathrm{PE}$ in the differential diagnosis [1]. The risk factors reported hereafter can be indications for the presence of pulmonary embolism:

- venous stasis;

- hypercoagulable states;

- immobilization;

- surgery and trauma;

- pregnancy;

- oral contraceptive and estrogen replacement;

- malignancy;

- hereditary factors resulting in a hypercoagulable state;

- acute medical illness;

- drug abuse (intravenous drugs);

- drug induced lupus anticoagulant;

- hemolytic anemias;

- heparin associated thrombocytopenia;

- homocystinemia;

- homocystinuria;

- hyperlipidemias;

- phenothiazines;

- thrombocytosis;

- varicose veins;

- venography;

- venous pacemaker;

- warfarin (first few days of therapy); and

- inflammatory bowel disease.

The PIOPED study listed also some indicators for PE [4]:

- travel lasting 4 hours or more in the past month;

- surgery within the last 3 months;

- malignancy, especially lung cancer;

- current or past history of thrombophlebitis;

- trauma in the lower extremities and pelvis during the past 3 months;

- smoking;

- central venous instrumentation within the past 3 months;

- stroke, paresis or paralysis;

\begin{tabular}{|c|c|}
\hline Symptom & Incidence \\
\hline Dyspnea & $73 \%$ \\
\hline Pleuritic chest pain & $66 \%$ \\
\hline Cough & $37 \%$ \\
\hline Hemoptysis & $13 \%$ \\
\hline
\end{tabular}

- prior pulmonary embolism;

- heart failure; and

- chronic obstructive pulmonary disease (COPD).

Among the biomarkers offering useful clinical information [5]:

- cardiac troponin levels may be elevated, particularly in patients with acute PE;

- plasma B-type natriuretic peptide was found elevated in patients with RV dysfunction from acute PE; and

- $\mathrm{D}$-dimer test indicates possible $\mathrm{PE}$; it has a sensitivity of $96-98 \%$, but the test alone is non specific, because it results positive also in case of cancer, infection, injury, and inflammation; therefore it has to be evaluated together with clinical signs and symptoms. Anyway the $\mathrm{D}$-dimer test is not predictive of DVT in stroke patients. Among the instrumental test helping the diagnosis, CT angiography has the greatest sensitivity and specificity for detecting emboli in the main, lobar, or segmental pulmonary arteries.

The echocardiogram is fundamental both to study the hemodynamic measures of RV and to guide the treatment of PE.

The diagnosis of pulmonary embolism should be suspected in patients with respiratory symptoms unexplained by an alternative diagnosis in presence of risk factors. In $\mathrm{pa}^{-}$ tients with recognized pulmonary embolism, the main physical signs are [1]:

- tachypnea (respiratory rate $>16 / \mathrm{min}$ );

- rales;

- accentuated second heart sound;

- tachycardia (heart rate > 100/min);

- fever (temperature $>37,8^{\circ} \mathrm{C}$ );

- diaphoresis;

- $\mathrm{S}_{3}$ or $\mathrm{S}_{4}$ gallop;

- clinical signs and symptoms suggesting thrombophlebitis;

- lower extremity edema;

- cardiac murmur; and

- cyanosis.
Table I. The PIOPED

study reported the following incidence of common symptoms of PE [4] 
Table II. Form filled for every patient admitted in the neurological ward. The reference guidelines are those used in Tuscany region [9]

BMI = Body Mass Index COPD $=$ Chronic Obstructive Pulmonary Disease; $\mathrm{NYHA}=$ New York Heart Association

*circle where appropriate 'lack of antithrombin, C protein, $\mathrm{S}$ protein, homozygosity for Leiden V factor or G20210A prothrombin or double heterozygous

sheterozygous for $\mathrm{V}$ Leiden factor or G20210A prothrombin

\begin{tabular}{|c|c|c|c|c|}
\hline \multicolumn{2}{|c|}{ Predisposing factors } & Points: & Factors related to the euent & Points* \\
\hline \multicolumn{2}{|c|}{$\begin{array}{l}\text { Personal history of venous } \\
\text { thromboembolism }\end{array}$} & 2.0 & $\begin{array}{l}\text { Hemiplegia or paraplegia from neurological } \\
\text { damage }\end{array}$ & 2.0 \\
\hline \multicolumn{2}{|c|}{ Congenital thrombophilia ${ }^{\circ}$} & 2.0 & Active cancer & 2.0 \\
\hline \multicolumn{2}{|c|}{ Antiphospholipid antibody } & 2.0 & $\begin{array}{l}\text { Respiratory failure with non-invasive } \\
\text { mechanical ventilation }\end{array}$ & 2.0 \\
\hline \multicolumn{2}{|c|}{ Staying in bed } & 1.5 & $\begin{array}{l}\text { Chemotherapy or radiotherapy or hormone } \\
\text { therapy }\end{array}$ & 2.0 \\
\hline \multicolumn{2}{|c|}{ Obesity (BMI > 30) } & 1.0 & Heart failure III-IV cl. NYHA & 2.0 \\
\hline \multicolumn{2}{|c|}{$\begin{array}{l}\text { Contraceptive pill or replacement } \\
\text { therapy after menopause }\end{array}$} & 1.0 & Acute infectious disease including sepsis & 2.0 \\
\hline \multicolumn{2}{|c|}{ Congenital thrombophilia ${ }^{\S}$} & 1.0 & COPD exacerbation & 1.0 \\
\hline \multicolumn{2}{|c|}{ Varicose vein } & 1.0 & Myocardial infarction & 1.0 \\
\hline \multicolumn{2}{|c|}{ Reduced mobility } & 1.0 & Minor surgery & 1.0 \\
\hline \multicolumn{2}{|c|}{$\begin{array}{l}\text { Familiar history of venous } \\
\text { thromboembolism }\end{array}$} & 1.0 & High risk surgery & 2.0 \\
\hline Age & $\begin{array}{c}40-60 \\
60-75 \\
>75\end{array}$ & $\begin{array}{l}0.5 \\
1.0 \\
1.5\end{array}$ & Chronic inflammatory bowel disease & 1.0 \\
\hline
\end{tabular}

\section{PATHOPHYSIOLOGY OF ACUTE PE}

In acute $\mathrm{PE}$ there are both respiratory and hemodynamic consequences. Acute respiratory consequences of $\mathrm{PE}$ include the following:

- increased alveolar dead space;

- hypoxemia; and

- hyperventilation.

Additional possible consequences include regional loss of surfactant and pulmonary infarction [1]. Arterial hypoxemia is frequently found in patients with acute embolism. The mechanism of hypoxemia includes ventilation-perfusion mismatch, intrapulmonary shunt, reduced cardiac output, and intracardiac shunt via a patent foramen ovale.

Pulmonary infarction is an uncommon consequence and is due to the bronchial arterial collateral circulation. $\mathrm{PE}$ reduces the area of the pulmonary vascular bed, thus causing an increment in pulmonary vascular resistance, which increases the right ventricular afterload. If the afterload is severely increased, the right ventricular failure happens. In addition, the humoral and reflex mechanism contribute to the pulmonary arterial constriction.

\section{DVT PROPHYLAXIS AND THERAPY OF PE IN ISCHEMIC STROKE}

$\mathrm{PE}$ is a potentially preventable complication after stroke $[6,7]$. The importance of thromboprophylaxis is relevant and is now considered one of the quality measures in a stroke unit [8]. In our department we use to fill a specific form for every patient admitted to neurological ward (Table II). This card with a risk stratification score includes all predisposing factors of the patient and the factors that led to hospitalization and is used for the evaluation of the risk of venous thromboembolism.

By adding the points, an individual score is obtained. The risk stratification is consequently made as indicated in Table III.

Prophylaxis is generally performed throughout the period of hospitalization and even beyond if reduced mobility persists, up to a maximum of 28 days.

In our ward, we prescribe Low-MolecularWeight Heparin (LMWH) according to the form reported in Tables II and III. We find it useful, however this isn't a validated score.

We report also the Padua Prediction Score [10] (Table IV), a validated score conceived by an Italian group and now spreading, also due to the inclusion in the American College of Chest Physicians guidelines about the prevention of VTE in nonsurgical patients [11]. It is deemed to be the best clinical model now available to assess the thrombotic risk in hospitalized patients.

National Institute for Health and Care Excellence (NICE) guidelines 2015 [12] for patients admitted for stroke recommend not to offer anti-embolism stockings for VTE prophylaxis to patients, but consider offering 
prophylactic-dose LMWH (or unfractionated heparin for patients with severe renal impairment or established renal failure) if:

- a diagnosis of hemorrhagic stroke has been excluded; and

- the risk of bleeding (hemorrhagic transformation of stroke or bleeding into another site) is assessed to be low.

Anti-embolism stockings are not recommended for ischemic stroke, but they are important and recommended in case of hemorrhagic stroke. The reason of this decision lies in the data from CLOT 3 study [13], showing that patients who have used intermittent pneumatic compression (IPC) up to six months after stroke have a more severe disability than patients treated with medical therapy alone. In our opinion, these data are inconclusive because it is important to distinguish between ischemic and hemorrhagic stroke in the trials and also to study patients similar in severity at the onset of stroke.

Unfractionated heparin (UFH) and LMWH reduce DVT after AIS; also intermittent pneumatic compression reduces the risk of DVT in AIS with immobility. The Italian clinical guidelines for ischemic stroke management recommend prophylactic LMWH or UFH to all immobilized patients with no contraindications. A low dose of LMWH or UFH seems to be effective and relatively safe as a prophylactic agent to prevent venous thromboembolism in patients with ischemic stroke. In these patients, the benefit-risk profile seems best for low-dose LMWH [14]. The duration of antithrombotic prophylaxis in almost all studies was 14 days or less. Since the occurrence of pulmonary embolism peaks at 2-4 weeks after stroke onset, it is possible that the duration of prophylaxis was in most of the cases too short to provide full benefit. In our experience, we recommended $\mathrm{LMWH}$ to all patients with ischemic stroke for 20 days at home.

Results from EXCLAIM study show that extended duration thromboprophylaxis with enoxaparin was associated with reduced venous thromboembolism risk and increased major bleeding in the subgroup of patients with ischemic stroke [15]. In patients who cannot receive anticoagulants for DVT prophylaxis, the use of aspirin and intermittent external compression devices are recommended. The use of intermittent external compression devices is recommended for treatment of patients who cannot receive anticoagulants.

\begin{tabular}{|c|c|l|}
\hline Individual score & Risk stratification & \multicolumn{1}{|c|}{ Prophylaxis } \\
\hline$<2.5$ & Low & No prophylaxis \\
$\geq 2.5$ & High & LMWH or fondaparinux $2.5 \mathrm{mg}$ \\
$>4$ & High & Consider adding also physical setting \\
\hline
\end{tabular}

The medical treatment of acute massive pulmonary embolism is fibrinolysis with alteplase.

The classification of patients with acute PE based on early mortality risk is an important prognostic assessment strategy [16]. For prediction of early (in hospital or 30-day) outcome in patients with acute $\mathrm{PE}$, both the PE-

Table III. Risk stratification for pulmonary embolism and need for thromboprophylaxis. The reference guidelines are those used in Tuscany region [9]

LMWH = Low-MolecularWeight Heparin

\begin{tabular}{|c|c|}
\hline Baseline features & Score \\
\hline Active cancer* & 3 \\
\hline Previous VTE (with the exclusion of superficial vein thrombosis) & 3 \\
\hline Reduced mobility ${ }^{\dagger}$ & 3 \\
\hline Already known thrombophilic condition ${ }^{\ddagger}$ & 3 \\
\hline Recent ( $\leq 1$ month) trauma and/or surgery & 2 \\
\hline Elderly age ( $\geq 70$ years) & 1 \\
\hline Heart and/or respiratory failure & 1 \\
\hline Acute myocardial infarction or ischemic stroke & 1 \\
\hline Acute infection and/or rheumatologic disorder & 1 \\
\hline Obesity (BMl $\geq 30$ ) & 1 \\
\hline Ongoing hormonal treatment & 1 \\
\hline
\end{tabular}

related risk and the patient's clinical status and comorbidities should be taken into consideration. At the stage of clinical suspicion of PE, hemodynamically unstable patients with shock or hypotension should immediately be identified at high-risk patients. They require an emergency diagnostic algorithm, if $\mathrm{PE}$ is confirmed, primary pharmacological reperfusion or alternatively surgical or interventional therapy. Patient without shock or hypotension are not at high risk of an adverse early outcome. Further risk stratification should be validated with a clinical prognostic score like Pulmonary Embolism Severity Index (PESI) or simplified Pulmonary Embolism Severity Index (sPESI), able to distinguish between intermediate and low risk. Around one-third of PE patients are at low risk of an early adverse outcome as indicated by PESI class I or II or a simplified PESI of 0. In these cases there are no therapeutic implications. On the other hand, patients in PESI class III-IV

\section{Table IV. Risk} assessment model (high risk of VTE: $\geq 4$ ) of the Padua Prediction Score [10]

BMI $=$ Body Mass Index; VTE $=$ Venous ThromboEmbolism

* Patients with local or distant metastases and/ or in whom chemotherapy or radiotherapy had been performed in the previous 6 months.

${ }^{\dagger}$ Bedrest with bathroom privileges (either due to patient's limitations or on physicians order) for at least 3 days.

${ }^{\ddagger}$ Carriage of defects of antithrombin, protein $\mathrm{C}$ or $\mathrm{S}$, factor V Leiden, G20210A prothrombin mutation, antiphospholipid syndrome. 
had a 30 -day mortality rate of up to $24,5 \%$ and those with a simplified PESI $\geq 1$ up to $11 \%$. Accordingly, normotensive patients in PESI class $\geq$ III or a simplified PESI $\geq 1$ are considered to constitute an intermediate-risk group. Within this category, further risk assessment should be considered, focusing on the status of the right ventricular (RV) in response to the $\mathrm{PE}$-induced acute pressure overload [17]. Patients with evidence of both RV dysfunction and elevated serum cardiac biomarker levels should be classified into an intermediate-high-risk category. In these cases, it is recommended to monitor hemodynamic parameters for initiation of reperfusion therapy. On the other hand, patients with normal RV and/or with normal cardiac biomarker levels belong to an intermediatelow-risk group.

The therapeutic strategies of pulmonary embolism depend on the risk stratification [16]. Primary reperfusion treatment, particularly systemic thrombolysis is the treatment of choice for patient with high-risk PE. In patients with contraindications to thrombolysis and in those in whom thrombolysis was not able to improve the hemodynamic status, surgical embolectomy is recommended. As an alternative to surgery, percutaneous catheter-directed treatment should be considered. On the other hand, for most case of acute PE without hemodynamic compromise, LMWH or fondaparinux, given subcutaneously at weight-adjusted doses without monitoring, is the treatment of choice unless there is severe renal dysfunction. In patients in intermediate-high risk group, systemic thrombolysis is not routinely recommended as primary treatment, but should be considered if clinical signs of hemodynamic decompensation appear. Surgical pulmonary embolectomy or percutaneous catheter-directed treatment may be considered as alternative, rescue procedures for patients with intermediate-high-risk $\mathrm{PE}$, in whom hemodynamic decompensation appears imminent and the anticipated bleeding risk under systemic thrombolysis is high. Anticoagulation is indicated in patients in intermediate-low-risk group. Patent foramen ovale (PFO) and related ischemic stroke are frequent in intermediate-risk PE. Considering the high risk of intracranial bleeding with thrombolysis in $\mathrm{PE}$, which may be partly due to hemorrhagic transformation of subclinical strokes, screening $\mathrm{PFO}$ should be considered in intermediate risk PE when thrombolytic treatment is discussed [18].
Finally, patients presenting with hemodynamic instability are usually treated with either thrombolytic therapy or pulmonary embolectomy followed by anticoagulation therapy, while most hemodinamically stable patients can be treated with anticoagulation alone. Anticoagulation medications include the following:

- unfractionated heparin;

- low molecular weight heparin;

- factor Xa inhibitors;

- fondaparinux; and

- warfarin.

Long-term anticoagulation is critical to the prevention of recurrence of DVT or PE, because even in fully anticoagulated patients, DVT and PE can often recur. The general consensus is that a significant reduction in recurrence is associated with 3-6 months of anticoagulation. In addition, previous ischemic stroke within 3 or 6 months is a contraindication to thrombolysis in ESC guidelines [16].

However, a study involving 145 patients with a stroke within 3 months who received thrombolysis for a further stroke did not show an increase in intracranial hemorrhage [19]. Condliffe and colleagues consider previous ischemic stroke not to be an absolute contraindication to thrombolysis, but at the moment there are no data to guide an acceptable timescale since the stroke [20].

Stroke guidelines advise delaying anticoagulation for 2 weeks post-ischemic stroke in patients with atrial fibrillation, but give discordant advice regarding anticoagulation for coexisting PE. UK stroke guidelines suggest anticoagulation for proximal DVT or PE, while American Heart Association guidelines do not recommend initial anticoagulation in patients with moderate to severe stroke $[21,22]$. The risk-benefit ratio for individual patients should be assessed; however the general approach is to anticoagulate all patients with a cerebral infarct and $\mathrm{PE}$. In patients with $\mathrm{PE}$ and a primary hemorrhagic stroke or recent significant hemorrhagic transformation, it is possible to consider inferior vena cava filter insertion and delayed anticoagulation [20]. Venous filter are usually placed in the infrarenal portion of the inferior vena cava. Venous filters are indicated in patient with acute PE who have absolute contraindications to anticoagulant drugs, and in patients with objectively confirmed recurrent $\mathrm{PE}$ despite adequate anticoagulation treatment. 
Key points

- Pulmonary embolism (PE) is a common and potentially lethal condition

- Patients with acute pulmonary embolism often present with dyspnea or chest pain

- It is often diagnosed with important delays, due to nonspecific symptoms, affecting a timely treatment

- Pulmonary embolism is not a disease in itself, but a complication of venous thromboembolism, most commonly deep vein thrombosis

- It is possible to prevent it after stroke with thromboprophylaxis

- The medical treatment of acute massive pulmonary embolism is fibrinolysis

- Patients presenting with hemodynamic instability are usually treated with either thrombolytic therapy or pulmonary embolectomy followed by anticoagulation therapy, while most hemodinamically stable patients can be treated with anticoagulation alone

- Long term anticoagulation is critical to the prevention of recurrence

Observational studies suggest that insertion of a venous filter might reduce PE-related mortality rates in the acute phase, benefit possibly coming at the cost of an increased risk of recurrence of VTE.
In conclusion, the management of these patients should be done after a thorough clinical assessment, taking into account the risks, benefits and local availability for every therapeutical option.

\section{REFERENCES}

1. Quellette DR, Kamangar N,Harrington A.Pulmonary Embolism. Medscape. Available at http:// emedicine.medscape.com/article/300901-overview (last accessed December 2015)

2. Liu LP, Zheng HG, Wang DZ, et al. Risk assessment of deep-vein thrombosis after acute stroke: a prospective study using clinical factors. CNS Neurosci Ther 2014; 20: 403-10; http:// dx.doi.org/10.1111/cns.12227

3. Pongmoragot J, Rabinstein AA, Nilanont Y, et al.; Investigators of Registry of Canadian Stroke Network (RCSN) and University of Toronto Stroke Program for Stroke Outcomes Research Canada (SORCan [www.sorcan.ca]) Working Group. Pulmonary embolism in ischemic stroke: clinical presentation, risk factors, and outcome. J Am Heart Assoc 2013; 2: e000372

4. Stein PD, Beemath A, Matta F, et al. Clinical characteristics of patients with acute pulmonary embolism: data from PIOPED II. Am J Med 2007; 120: 871-9; http://dx.doi.org/10.1016/j. amjmed.2007.03.024

5. Pelliccia F, Schiariti M, Terzano C, et al. Treatment of acute pulmonary embolism: update on newer pharmacologic and interventional strategies. Biomed Res Int 2014; 2014: 410341; http:// dx.doi.org/10.1155/2014/410341

6. Torbicki A, Perrier A, Konstantinides S, et al.; ESC Committee for Practice Guidelines (CPG). Guidelines on the diagnosis and management of acute pulmonary embolism: the Task Force for the Diagnosis and Management of Acute Pulmonary Embolism of the European Society of Cardiology (ESC). Eur Heart J2008; 29:2276-315; http://dx.doi.org/10.1093/eurheartj/ehn310

7. Piazza G, Goldhaber SZ. Acute pulmonary embolism: part II: treatment and prophylaxis. Circulation 2006; 114: e42-7; http://dx.doi.org/10.1161/CIRCULATIONAHA.106.620880

8. Gensini GF, Zaninelli A, Ricci S, et al. SPREAD, Stroke Prevention And Educational Awareness Diffusion, VII Edizione, Ictus cerebrale: linee guida italiane di prevenzione e trattamento. Raccomandazioni e sintesi. 14 marzo 2012. Available at http://www.siapav.it/pdf/SPREAD\%20 2012.pdf (last accessed December 2015)

9. Regione Toscana. Linee guida per la profilassi del tromboembolismo venoso nei pazienti ospedalizzati. SNLG Regioni, 2015. Available at http://www.snlg-iss.it/cms/files/LG_Toscana_ TEV.pdf (last accessed December 2015)

10. Barbar S, Noventa F, Rossetto V, et al. A risk assessment model for the identification of hospitalized medical patients at risk for venous thromboembolism: the Padua Prediction Score. J Thromb Haemost 2010; 8: 2450-7; http://dx.doi.org/10.1111/j.1538-7836.2010.04044.x 
11. Kahn SR, Lim W,Dunn AS, et al.; American College of Chest Physicians. Prevention of VTE in nonsurgical patients: Antithrombotic Therapy and Prevention of Thrombosis, 9th ed: American College of Chest Physicians Evidence-Based Clinical Practice Guidelines. Chest 2012; 141 (2 Suppl): e195S-226S

12. National Institute for Health and Care Excellence. Stroke and transient ischaemic attack in over 16s: diagnosis and initial management. Available at https://www.nice.org.uk/guidance/ cg68 (last accessed December 2015)

13. CLOTS (Clots in Legs Or sTockings after Stroke) Trials Collaboration, Dennis M, Sandercock $\mathrm{P}$, et al. Effectiveness of intermittent pneumatic compression in reduction of risk of deep vein thrombosis in patients who have had a stroke (CLOTS 3): a multicentre randomised controlled trial. Lancet 2013; 382: 516-24; http://dx.doi.org/10.1016/S0140-6736(13)61050-8

14. Wells PS, Forgie MA, Rodger MA. Treatment of venous thromboembolism. JAMA 2014; 311: 717-28; http://dx.doi.org/10.1001/jama.2014.65; http://dx.doi.org/10.1001/jama.2014.6123

15. Turpie AG,Hull RD, Schellong SM, et al.; EXCLAIM Investigators. Venous thromboembolism risk in ischemic stroke patients receiving extended-duration enoxaparin prophylaxis: results from the EXCLAIM study. Stroke 2013; 44: 249-51; http://dx.doi.org/10.1161/ STROKEAHA.112.659797

16. Konstantinides SV.2014 ESC Guidelines on the diagnosis and management of acute pulmonary embolism. Eur Heart J 2014; 35: 3145-6

17. Konstantinides SV, Torbicki A. Management of pulmonary embolism: recent evidence and the new European guidelines. Eur Respir J 2014; 44: 1385-90; http://dx.doi. org/10.1183/09031936.00180414

18. Doyen D, Castellani M, Moceri P, et al. Patent foramen ovale and stroke in intermediate-risk pulmonary embolism. Chest 2014; 146: 967-73; http://dx.doi.org/10.1378/chest.14-0100

19. Karlinski M, Kobayashi A, Mikulik R, et al. Intravenous Alteplase in ischemic stroke patients not fully adhering to the current drug license in Central and Eastern Europe. Int J Stroke 2012; 7: 615-22; http://dx.doi.org/10.1111/j.1747-4949.2011.00733.x

20. Condliffe R, Elliot CA, Hughes RJ, et al. Management dilemmas in acute pulmonary embolism. Thorax 2014; 69: 174-80; http://dx.doi.org/10.1136/thoraxjnl-2013-204667

21. Royal College of Physicians. National clinical guideline for stroke. September 2012, fourth edition. Available at https://www.rcplondon.ac.uk/file/1299/download?token=mcyQFjEq (last aaccessed December 2015)

22. Jauch EC, Saver JL, Adams HP, et al; American Heart Association Stroke Council; Council on Cardiovascular Nursing; Council on Peripheral Vascular Disease; Council on Clinical Cardiology. Guidelines for the early management of patients with acute ischemic stroke: a guideline for healthcare professionals from the American Heart Association/American Stroke Association. Stroke 2013; 44: 870-947; http://dx.doi.org/10.1161/STR.0b013e318284056a 